\title{
A double attack on pain
}

Increasing evidence suggests that G protein-coupled receptors (GPCRs) can exist as heteromeric complexes with pharmacological properties that differ from the individual GPCRs. Two recent studies published in PNAS show that ligands targeting a heteromer consisting of the $\mu$-opioid receptor (the target of the analgesic morphine) and a second GPCR could overcome some of the limitations of morphine.

Akgün and colleagues focused on the $\mu$-opioid receptor and the metabotropic glutamate receptor 5 (mGluR5). These two receptors are co-expressed in neurons and potentially exist as heteromers, and mGluR5 antagonists are known to improve the analgesic effects of morphine.

The authors designed a series of bivalent ligands that contained

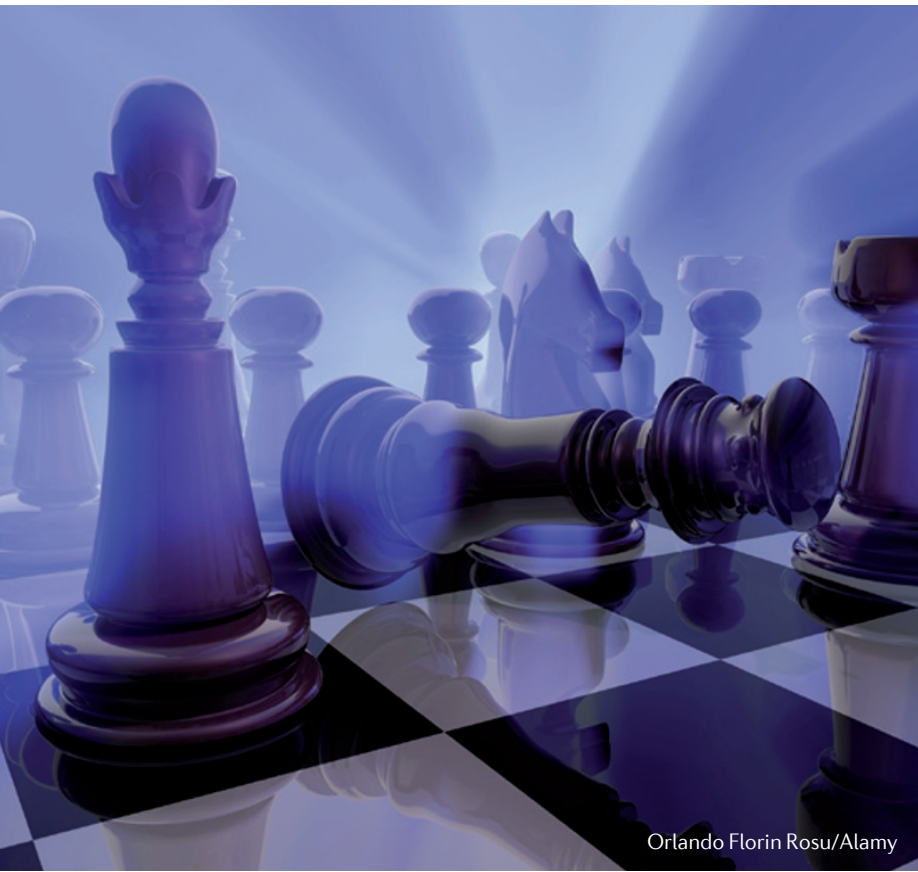

pharmacophores of an mGluR5 antagonist and a $\mu$-opioid receptor agonist linked with spacer structures. The compounds were then tested in mouse models of inflammatory pain and bone cancer pain. When given by intrathecal injection, the most potent compound (with a spacer length of 22 atoms, named MMG22) had $\mathrm{ED}_{50}$ (half-maximal effective dose) values of 7.7-9.0 fmol per mouse in inflammatory pain models, and an $\mathrm{ED}_{50}$ value of $3.8 \mathrm{fmol}$ per mouse in the bone cancer pain model.

Notably, MMG22 was 40,000 times more potent than the combination of the monovalent compounds. In addition, the compound was much less potent when given by intracerebroventricular injection or when given intrathecally to mice without inflammatory pain, which suggests that the target of the compound is upregulated in the spinal cord during pain.

Importantly, no tolerance to the analgesic effects of MMG22 was observed, and it did not induce respiratory depression (two side effects of morphine). So, this study suggests that bivalent ligands that target the $\mu$-opioid receptor and mGluR5 could offer a new treatment option for pain.

Gomes and colleagues focused on the $\mu$-opioid receptor and the $\delta$-opioid receptor, which can also exist as heteromeric complexes. Indeed, $\delta$-opioid receptor antagonists have been shown to potentiate the analgesic effects of morphine.

To identify agonists that specifically target the $\mu$-opioid- $\delta$-opioid receptor complex, they performed a screen of $\sim 335,000$ compounds from the US molecular libraries probe collection in cell lines expressing both receptors or either receptor. Following additional screens for potency, heteromer selectivity and novelty of the chemical scaffold, one compound - named CYM51010 (PubChem Compound ID: 23723457) - was chosen for further investigation.

The authors then measured the antinociceptive effects of CYM51010 in a mouse model of thermal pain. Subcutaneous administration of CYM51010 produced antinociception that was comparable to that produced by morphine ( $a n \mathrm{ED}_{50}$ of $\sim 5 \mathrm{mg}$ per $\mathrm{kg}$ ), but was notably associated with less antinociceptive tolerance. The effects of CYM51010 were partially reversed by a $\mu$-opioid receptor antagonist and substantially reversed by an antibody specific for the $\mu$-opioid- $\delta$-opioid receptor heteromer; this suggests that most of the analgesic effects of CYM51010 were attributable to its effects on the heteromer.

The authors suggest that CYM51010 could act as a chemical scaffold - which contains one pharmacophore - for the further development of ligands that act at the $\mu$-opioid- $\delta$-opioid receptor heteromer.

Together, these studies suggest that targeting the $\mu$-opioid receptor in complex with another GPCR could lead to the development of more effective analgesics.

Charlotte Harrison

ORIGINAL RESEARCH PAPERS Akgün, E. et al. Ligands that interact with putative MOR-mGluR5 heteromer in mice with inflammatory pain produce potent antinociception. Proc. Natl Acad. Sci. USA 110, 11595-11599 (2013)|Gomes, I. et al. Identification of a $\mu-\delta$ opioid receptor heteromerbiased agonist with antinociceptive activity. Proc. Natl Acad. Sci. USA 110, 12072-12077 (2013) 